\title{
An application of Cooperative Game Theory to Distributed Control ${ }^{\star}$
}

\author{
J. M. Maestre* D. Muñoz de la Peña* A. Jiménez Losada** \\ E. Algaba Durán ${ }^{* *}$ E. F. Camacho* \\ * Dep. of Systems and Automation Engineering, University of Seville, \\ Spain (e-mail: \{pepemaestre, davidmps, eduardo\}@cartuja.us.es). \\ ** Dep. of Applied Math II, University of Seville, Spain (e-mail: \\ hispan@esi.us.es,ealgaba@us.es)
}

\begin{abstract}
In this paper we propose to study the underlying properties of a given distributed control scheme in which a set of agents switch between different communication strategies that define which network links are used in order to regulate to the origin a set of unconstrained linear systems. The problems of how to decide the time-varying communication strategy, share the benefits/costs and detect which are the most critical links in the network are solved using tools from game theory. The proposed scheme is demonstrated through a simulation example.
\end{abstract}

\section{INTRODUCTION}

Over the last few years there has been a growing interest of the control community in the research of distributed control systems. The basic idea is to divide the overall system into several smaller subsystems, each controlled by a different local controller or agent which may or may not share information with the rest. Examples of these situations are large scale systems or networked systems such as traffic, water or power networks; see R. R. Negenborn and Hellendoorn. [2006]. In addition, the proliferation of wireless actuator/sensor devices and their application to enhance the possibilities of advanced control systems also bring new challenges; see Neumann [2007]. Issues such as the communicational burden of the different distributed architectures play a very important role. For example, the battery life of motes depend specially on the time the radio is on and the number of messages sent. Although there are works in the literature that deal with the dynamics induced by the communication network (such as time-varying delays and data losses)(see for example de Wit [2006]) or the specific distributed control algorithm implemented by the local controllers(see for example R. R. Negenborn and Hellendoorn. [2006] or Venkat [2006]), in general, there are few results that deal with the underlying properties of a given distributed control architecture. Moreover, these properties may be dynamical and distributed control policies should be flexible enough to adapt to the possible changes.

Decentralized and distributed control schemes often assume that the centralized system is partitioned into a fixed set of neighborhoods In Alessio and Bemporad [2007] a decentralized a model predictive control scheme and a sufficient criterion for analyzing a posteriori the asymptotic stability of the corresponding closed-loop system were presented. This work is enhanced by Alessio and Bemporad [2008] to take into account possible data losses. Liu et al. [2008] proposed a decentralized control architecture for nonlinear systems with continuous and asynchronous measurements. Magni and Scattolini [2006] proposes a predictive controller under the main assumptions that the system is nonlinear, discrete-time and no information is exchanged between local controllers. The stability of this class of systems was studied by Raimondo et al. [2007].

In general, the composition of these neighborhoods is assumed static, that is, the possibility of time varying neighborhoods is not considered. In addition, there are other interesting questions that are not addressed such as which elements of a given distributed control system are more critical. Motivated by these issues, in this paper we study the underlying properties of a given distributed control scheme using tools from game theory. From a mathematical point of view, game theory is an appropriate framework to study all the phenomena that arise from the mutual interaction of agents that take their decisions alone or in cooperation; see Myerson [1997]. Actually, important game theory concepts such as Pareto optimality and Nash equilibrium have been used to study the properties of control schemes, see for instance the work by Venkat et al. [2005] or by Maestre et al. [2010]. In this paper we use tools from coalitional game theory Saad et al. [2009], a branch of game theory which studies the role played by communication networks in situations of conflict.

In particular, we focus on the following three different problems:

- Given a communication network, do all the links have to be enabled all the time?

- Do all the links and the agents have the same relevance in a networked control system?

- When several agents cooperate to reach a certain objective, do they have to share equally the costs or benefits of the cooperation? 


\section{PROBLEM FORMULATION}

In this paper we consider discrete time linear systems that can be partitioned in $N$ subsystems whose dynamics are given by the following model:

$$
\begin{aligned}
& x_{i}(t+1)=A_{i i} x_{i}(t)+B_{i i} u_{i}(t)+d_{i}(t), \\
& d_{i}(t)=\sum_{j \neq i} A_{i j} x_{j}(t)+\sum_{j \neq i} B_{i j} u_{j}(t),
\end{aligned}
$$

where $x_{i} \in \mathbb{R}\left(q_{i}\right)^{1}$ and $u_{i} \in \mathbb{R}\left(r_{i}\right)$ with $i=1, \ldots, N$ are the states and inputs of each subsystem respectively. The variable $d_{i}$ is the influence of the neighbors' states and inputs in the update of $x_{i}$.

Each subsystem is controlled by a different agent that has access only to its state $x_{i}$ and decides at each sample time the value of its corresponding input $u_{i}$. In addition, the agents can communicate through a network which is defined as a graph $(N, L)$, where $L$ is the set of edges $L \subseteq L^{N}=\{\{i, j\} \mid\{i, j\} \subseteq N, i \neq j\}$ which define the available communication links between the agents. Note that under this definition $i j$ and $j i$ represent the same link. In this paper we assume that the necessary and sufficient condition for any two agents to communicate is that they are at least indirectly connected by the network, that is, there exists a path of active links that connect them. Additionally, we will assume that the communication has a cost. In particular, we consider that each link has a fixed cost $c>0$ associated to its use.

The control objective is to regulate the state of all the subsystems to the origin while minimizing a cost that depends on the state and input trajectories and the communications. The stage cost of each agent is defined as follows

$$
\ell_{i}(t)=x_{i}^{T}(t) Q_{i} x_{i}(t)+u_{i}^{T}(t) R_{i} u_{i}(t),
$$

where $Q_{i} \in \mathbb{R}\left(q_{i} \times q_{i}\right)$ and $R_{i} \in \mathbb{R}\left(r_{i} \times r_{i}\right)$ are constant weighting matrices.

The communicational costs depend on the number of links that are being used. Given a network $(N, L)$, not all the links $L$ have to be used all the time. We define a network mode as a subset of links $A$ in $L$, composed only by those links which are enabled. Any link $l \notin A$ is considered to be disabled and, thus, absent. In the following section, we present a distributed control scheme that decides which network mode $A$ has to be active based on an analysis of the trade-off between control performance and communicational costs

\section{DISTRIBUTED CONTROL ALGORITHM}

In this section we present a distributed control scheme that at each sampling time, implements a certain communication strategy defined by a network mode $A$. The communication strategy $A$ is chosen every $D$ sample times. To this end, the agents must broadcast their state and take a decision about the communication strategy that will be used in the next $D$ time steps. This leads to a double sample rate control system. As a result of this policy, the agents are separated into separated groups $C$ that are able to communicate defined as communication components.

1 With a little abuse of notation, we will use the notation $\mathbb{R}(n)$ to denote $\mathbb{R}^{n}$.
We will denote by $N / A$ the set of all communication components in which the set $N$ is partitioned. Note that $\bigcup_{i \in N / A} C_{i}=N$ and $C_{i} \bigcap C_{j}=0$ for all $i \neq j$. $\forall i \in N / A$

We assume that at each sampling time each communication component $C \subseteq N / A$ implements a linear controller based on all the available state information

$$
u_{C}=K_{C}^{A} x_{C},
$$

where $u_{C} \in \mathbb{R}\left(\sum_{i \in C} r_{i}\right)$ is the input of a given communication component defined as $u_{C}=\left\{u_{i}\right\}_{i \in C}, x_{C} \in$ $\left.\mathbb{R}^{(} \sum_{i \in C} q_{i}\right)$ is the the state of a given communication component defined as $x_{C}=\left\{x_{i}\right\}_{i \in C}$ and $K_{C}^{A}$ is the matrix which defines the controller implemented by the communication component $C$ for the network mode $A$. Note that we have dropped the time dependence of $u_{C}$ and $x_{C}$ in order to simplify the notation.

The set of the matrices $K_{C}^{A}$ define a centralized linear controller for the global system. This controller is characterized by the absence of communication between agents that belong to different communication components. Thus, the equivalent centralized control law is given by

$$
u=K_{A} x,
$$

where $\left.u \in \mathbb{R}^{(} \sum_{i \in N} r_{i}\right)$ is the input of the centralized system defined as $u=\left\{u_{i}\right\}_{i \in N}, x \in \mathbb{R}\left(\sum_{i \in N} q_{i}\right)$ is the the state of the centralized system defined as $x=\left\{x_{i}\right\}_{i \in N}$ and $K_{A}$ is the matrix which defines the centralized controller implemented for the network mode $A$.

We assume that there exists a quadratic function that satisfies

$$
x^{T} P_{A} x \geq \sum_{j \in N} \sum_{k=0, . ., \infty} \ell_{j}(k),
$$

that is, $P_{A}$ is a weight matrix that provides an upper bound of the cost to infinity of the centralized system in closed-loop with the controller $u=K_{A} x$ starting from the initial state $x(0)=x$. The calculation details of the matrix $P_{A}$ is presented in section 5 .

Once the sets of matrices $P_{A}$ and $K_{A}$ are defined, the function $r(A, x)$ is defined as follows:

$$
r(A, x)=x^{T} P_{A} x+c|L(A)|, \forall A \subseteq L,
$$

where $|L(A)|$ stands for the number of active links in the network mode $A$. This function assigns a value to each communication mode $A$ based on the upper bound of the cost-to-go of all the communication components in the network and the corresponding communication costs. The decision about what communication strategy should be used during the next $D$ samples is made minimizing $r(A, x)$ over $A$.

Algorithm 1. At each sample time, the proposed distributed control scheme is implemented as follows:

(1) If the sample time is a multiple of $D$, all the agents broadcast their state and calculate which is the network mode $A$ that minimizes the function $r(A, x)$. Otherwise, each agent sends his state only to those agents that belong to his communication component.

(2) Each agent uses the state information received in order to update its control action using its corresponding communication component feedback matrix $K_{A}^{C}$. Globally, this implies that the linear controller $u=K_{A} x$ is applied. 
Remark: The matrix $K_{A}$ takes into account the communications constraints in $A$. For example, if the $i$-th element of $u$ and the $j$-th element of $x$ belong to different communication components, then $K_{A}(i, j)=0$; that is, the $i$-th input does not depend on the $j$-th state. For the particular case in which each communication component is composed by systems with consecutive numeration in the set $N$, then $K_{A}=\operatorname{diag}\left(K_{C_{1}}^{A}, K_{C_{2}}^{A}, \ldots\right)$.

Remark: All the matrices $K_{C}^{A}$ have to be designed so that $K_{A}$ guarantees closed-loop stability for the centralized system. If a given mode is not able to stabilize the system, then it is not taken into account.

Remark: The on-line implementation of the algorithm may be simplified by limiting the possible network modes $A$ that are considered, for example assuming that every $D$ sampling times only on link can be activated or deactivated.

\section{AN ANALYSIS OF THE RELEVANCE OF AGENTS AND LINKS}

In this section we answer the second and third questions that we made in the introduction. In the previous section we presented a control scheme to switch dynamically the state of the communication links based on the function $r(A, x)$. The presented control problem is mathematically equivalent to a cooperative game. Cooperative game theory studies situations of mutual interaction between a set of players which can negotiate among them and commit themselves to follow common binding strategies. As a result of the bargaining process, the set of players might be divided into several subsets called coalitions. The role of game theory is to study which coalitions of agents should be formed and to analyze how the costs or benefits from cooperation should be distributed among the members of a coalition.

In its most basic form, a cooperative game is defined only with two elements, a set $N$ of players and a characteristic function $v$ that assigns a value to each of the possible coalitions $S$ of agents. A coalition $S$ is defined as a subset of $N$. In case that the coalition is formed by all the players in $N$, the term grand coalition is used. The function $v(S)$ represents the value of the coalition, that is, the costs or benefits of reaching the common goal without the assistance of the agents that are not present in the coalition.

The definition of a cooperative game requires the exhaustive evaluation of the function $v$ for each possible coalition of players $S$. This description provides too much information for a simple analysis of the relevance of the agents and the links. All this information can be synthesized by means of a payoff rule, which is a mathematical tool which provides the individual outcomes of the game, that is, a payoff vector that specifies the benefit or cost that each player may reasonably expect from the game. Mathematically, a payoff or allocation vector is defined as $o=\left(o_{i}\right)_{i \in N} \in \mathbb{R}(N)$ and specifies for each player $i$ the profit or cost $o_{i}$ when he cooperates with other players. There are many different payoff rules in the literature. In this paper we will use the Shapley value, which is the only allocation rule $\gamma(N, v)$ that verifies the efficiency, additivity and symmetry properties (see Myerson [1997]). The Shapley value assigns a value $\gamma_{i}(N, v)$ to each agent $i \in N$ in a cooperative game whose value function is given by $v$. The Shapley value of a game is defined as

$\gamma_{i}(N, v)=\sum_{S \subseteq N \backslash\{i\}} \frac{|S| !(N-|S|-1) !}{N !}(v(S \cup\{i\})-v(S))$.

From a centralized point of view, the Shapley value of the game $(N, v)$ is the payoff vector that gives to each agent his expected marginal contribution to a random coalition.

We propose to use these ideas from cooperative game theory for the analysis of the relevance of the different agents and links of a distributed control scheme. To this end, it is necessary to extend the definition of cooperative game so that both the network and the communicational costs are considered. Thus, we define a cost-extended communication situation $H$ as as the tuple $(N, v, L, c)$. We already know the components of the tuple: $N$ is the set of players, $v$ is the characteristic function of the game, $L$ is the sets of links that compose the network and $c$ is the communicational cost associated to the use of a link. Together with the Shapley value, cost-extended communication situations allow one to study several inherent properties of the agents and the network. In the next subsections we show how to determine what agent and link play the most important role at a given time.

\subsection{Link analysis}

When modeling a distributing control problem as a cooperative game, the most natural way to proceed is to assume that the control agents are the players of the game. Nevertheless, it is possible to change the focus to links instead of agents, just as it is proposed in Borm et al. [1992]. Thus, the gains or costs from cooperation are attributed to communication links, which lead us to define a cost-extended "link game" as a tuple $\left(L, r^{H}\right)$ associated to the cost-extended communication situation $H$. The characteristic function for this game is defined as

$$
r^{H}(A)=\sum_{C \in N / A} v(C)+c|L(A)|, \forall A \subseteq L,
$$

which is defined for all the possible subsets $A$ of links contained in the original network $L$. In the characteristic function of the link game defined by equation (4), the grand coalition is divided into its communication components and its value is the sum of the values of the corresponding components and the cost of the links that are employed for the communication defined by the set $A$.

We propose to use the function $r(A, x)$ defined in equation (3) to define the link game characteristic function, that is, $r^{H}(A)=r(A, x)$. Therefore, given a state $x$, it is possible to construct the link game and a qualitative and quantitative analysis of the relevance of the links in the game may be obtained from the corresponding Shapley value $\gamma\left(L, r^{H}\right)$. Each component of this vector represents the cost of a given link for the system when the state is $x$. In other words, the lower value a link has, the higher utility it has for the system. 


\subsection{Agent analysis}

In this case the players of the game are the different agents. Let us consider a cooperative game $\left(N, w^{H}\right)$ defined by characteristic function $w^{H}(S)$ which assigns to each coalition the following cost

$$
w^{H}(S)=\sum_{C \in S / L} v(C)+c|L(S)|, \forall S \subseteq N,
$$

where $|L(S)|$ is the number of links that are used by the coalition $S$ and $c$ is the link cost. We denote this game as the "agent game". Note that according to (5) the value of a coalition $S$ is the sum of the values of its members separated into their corresponding communication components.

A qualitative and quantitative analysis of the relevance of the agents of the system can be obtained from the Shapley value $\gamma\left(N, w^{H}\right)$ of the corresponding agent game. To build such game it is necessary to define the characteristic function that assigns a value to each coalition $S$ of agents for a given network $L$. To this end, it is not possible to use the controllers defined for each communication component for a given network mode $A$ because those controllers take into account the particular communication constraints of network mode $A$. For this reason, for each communication component of $L$, we need a controller $K_{C}$ and a weight matrix $P_{C}$ such that

$$
x_{C}^{T} P_{C} x_{C} \geq \sum_{j \in C} \sum_{k=0, . ., \infty} \ell_{j}(k)
$$

where $x_{c}$ is obtained from the current state $x$ and is composed of the states of all the subsystems that belong to $\mathrm{C} ; P_{C}$ is a weight matrix that provides an upper bound for the cost to infinity of the systems that belong to $C$ in closed-loop with the controller $u_{C}(t)=K_{C} x_{C}(t)$ starting from the initial state $x_{C}(0)=x_{C}$ assuming that all the inputs and states that belong to agents outside the coalition are zero. This assumption allows only to calculate a simple approximation of the Shapley value of the agent game. Most conservative choices could have been made, for example the agents outside of the coalition could have been considered as disturbances and then a min-max approach used. The details about the calculation of the controller can be found in section 5 .

Then, the characteristic function of the agent game that defines the utility of a coalition $S$ is defined as

$$
w^{H}(S)=\sum_{C \in S / L} x_{C}^{T} P_{C} x_{C}+c|L(S)|, \forall S \subseteq N .
$$

The Shapley value of the game $\left(N, v^{H}\right)$ is known as the Myerson value of the game and provides concise information about the relevance of all the agents in the game. The lower the value the is, the more relevant role the agent has in the game. It is important to remark that the Shapley value of the agent game, as it is defined, does not have any physical meaning.

\section{CONTROLLER DESIGN PROCEDURE}

In this section we present a method to design all the matrices that define the controllers and their corresponding upper bounds on the cost. To this end, we provide two theorems, one to design the matrices that define each communication mode, and another one to obtain the matrices that define each possible coalition.

Theorem 1. Let $A \in L$ be a set of active links in a distributed control system. The dynamics of the whole system are given by $A_{N}=\left\{A_{i j}\right\}, \forall i, j \in N$ and $B_{N}=$ $\left\{B_{i j}\right\}, \forall i, j \in N$ and its stage cost defined by $Q_{N}=$ $\operatorname{diag}\left(Q_{i}\right)$ and $R_{N}=\operatorname{diag}\left(R_{i}\right), \forall i \in N$. If there exist matrices $W_{N}=\left\{W_{i j}\right\}, \forall i, j \in N$, where $W_{i, j} \in \mathbb{R}\left(q_{i} \times q_{j}\right)$, and $Y_{N}=\left\{Y_{i j}\right\}, \forall i, j \in N$, where $Y_{i, j} \in \mathbb{R}\left(r_{i} \times q_{j}\right)$, such that the following constraints are satisfied

$$
\left[\begin{array}{cccc}
W_{N} & W_{N} A_{N}^{T}+Y_{N}^{T} B_{N}^{T} & W_{N} Q_{N}^{1 / 2} & Y_{N}^{T} R_{N}^{1 / 2} \\
A_{N} W_{N}+B_{N} Y_{N} & W_{N} & 0 & 0 \\
Q_{N}^{1 / 2} W_{N} & 0 & I & 0 \\
R_{N}^{1 / 2} Y_{N} & 0 & 0 & I
\end{array}\right]>0
$$

s.t.

$W_{i j}=0, Y_{i j}=0 \forall i, j$ such that $x_{i} \in C, x_{j} \notin C$

then matrices $P_{A}=W_{N}^{-1}$ and $K_{A}=Y_{N} W_{N}^{-1}$ satisfy (2), all the communication constraints imposed by the network mode $A$ and stabilize the whole system.

In the agent game the approximation of the cost of the communication component $C$ is based on the cost to infinity given by the $x_{C}^{T} P_{C} x_{C}$. This upper bound is calculated assuming that the rest of the agents states and inputs are zero. The agent game only provides grounds for distributing the benefits or costs between the agents during the game and the resulting feedback gains are never used to control the system, so it is not necessary to impose centralized stability as a requirement. The following theorem presents an LMI constraint that can be used to solve this design problem.

Theorem 2. Let $C \in S / L$ be a set of independent communication components for a given communication situation $H$ whose dynamics are given by $A_{C}=\left\{A_{i j}\right\}, \forall i, j \in C$ and $B_{C}=\left\{B_{i j}\right\}, \forall i, j \in C$ and its stage cost defined by $Q_{C}=\operatorname{diag}\left(Q_{i}\right)$ and $R_{C}=\operatorname{diag}\left(R_{i}\right), \forall i \in C$. If there exist matrices $W_{C}$ and $Y_{C}$ such that the following constraint is satisfied

$$
\left[\begin{array}{cccc}
W_{C} & W_{C} A_{C}^{T}+Y_{C}^{T} B_{C}^{T} & W_{C} Q_{C}^{1 / 2} & Y_{C}^{T} R_{C}^{1 / 2} \\
A_{C} W+B_{C} Y & W_{C} & 0 & 0 \\
Q_{C}^{1 / 2} W_{C} & 0 & I & 0 \\
R_{C}^{1 / 2} Y_{C} & 0 & 0 & I
\end{array}\right]>0
$$

then matrices $P_{C}=W_{C}^{-1}$ and $K_{C}=Y_{C} W_{C}^{-1}$ satisfy (6) and stabilize the states of $C$ assuming that the state and inputs that do not belong to that communication component are zero.

Both theorems can be proved using standard techniques based on the application of Schur's complement.

\section{SIMULATION RESULTS}

In this section we show an academic example that illustrates the techniques proposed in the paper. The system is shown in figure 1 . The agents can communicate with a network defined by four links. We will refer to the agents using arabic numbers and to the links using roman letters. In order to implement the proposed control scheme, matrices $K_{A}$ and $P_{A}$ have to be designed for each of the possible modes. Given that there are four links, there are 


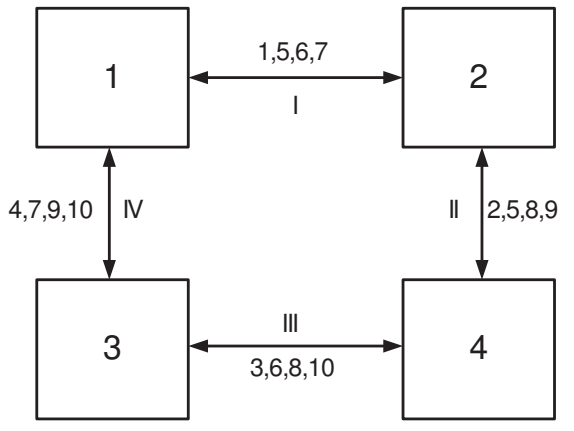

11-14: $3 / 4$ links 15: 4 links

Fig. 1. Links enabled in each mode.

16 possible network modes which have been numbered from 0 to 15 . The networks modes for which a link is enabled are shown next to it in figure 1.

The matrices that define the subsystem dynamics are the following:

$$
\begin{aligned}
& A_{11}=\left[\begin{array}{ll}
1 & 0.8 \\
0 & 0.7
\end{array}\right] B_{11}=\left[\begin{array}{l}
0 \\
1
\end{array}\right] A_{22}=\left[\begin{array}{ll}
1 & 0.6 \\
0 & 0.7
\end{array}\right] B_{22}=\left[\begin{array}{l}
0 \\
1
\end{array}\right] \\
& A_{33}=\left[\begin{array}{ll}
1 & 0.9 \\
0 & 0.8
\end{array}\right] \quad B_{33}=\left[\begin{array}{l}
0 \\
1
\end{array}\right] A_{44}=\left[\begin{array}{ll}
1 & 0.8 \\
0 & 0.5
\end{array}\right] \quad B_{44}=\left[\begin{array}{l}
0 \\
1
\end{array}\right] \\
& A_{i j}=\left[\begin{array}{ll}
0 & 0 \\
0 & 0
\end{array}\right] B_{i j}=\left[\begin{array}{c}
0 \\
0.15
\end{array}\right] \forall i \neq j
\end{aligned}
$$

where $x_{i} \in \mathbb{R}(2)$ with $i \in\{1, . ., 4\}$ are the states of each subsystem and $u_{i} \in \mathbb{R}(1)$ with $i \in\{1, \ldots, 4\}$ are the corresponding inputs. The stage costs $\ell_{i}$ of all the subsystems are defined by matrices $Q_{i}=\operatorname{diag}(1,1), R_{i}=$ 1 with $i=\{1,2,3,4\}$.

\subsection{Distributed control scheme}

For each mode, a different LMI problem designed according to Theorem 1 have been solved to obtain the corresponding matrices $K_{A}$ and $P_{A}$ using Matlab's LMI toolbox. For example, for mode 4 , which corresponds to the case in which agents 1 and 3 communicate and coordinate their actions. The resulting matrices are:

$$
\begin{gathered}
K_{4}^{T}=\left[\begin{array}{ccccc}
-0.25 & 0.00 & 0.02 & 0.00 \\
-0.53 & 0.00 & 0.06 & 0.00 \\
0.00 & -0.26 & 0.00 & 0.00 \\
0.00 & -0.45 & 0.00 & 0.00 \\
0.01 & 0.00 & -0.23 & 0.00 \\
0.05 & 0.00 & -0.63 & 0.00 \\
0.00 & 0.00 & 0.00 & -0.27 \\
0.00 & 0.00 & 0.00 & -0.43
\end{array}\right] \\
P_{4}=\left[\begin{array}{cccccccc}
4.56 & 5 & 0 & 0 & -0.36 & -1.1 & 0 & 0 \\
5 & 9.61 & 0 & 0 & -0.8 & -2.48 & 0 & 0 \\
0 & 0 & 5.48 & 5.14 & 0 & 0 & 0 & 0 \\
0 & 0 & 5.14 & 8.34 & 0 & 0 & 0 & 0 \\
-0.36 & -0.8 & 0 & 0 & 4.17 & 5.08 & 0 & 0 \\
-1.1 & -2.48 & 0 & 0 & 5.08 & 11.69 & 0 & 0 \\
0 & 0 & 0 & 0 & 0 & 0 & 5.37 & 5.44 \\
0 & 0 & 0 & 0 & 0 & 0 & 5.44 & 8.40
\end{array}\right]
\end{gathered}
$$

It can be seen that $K_{A}$ satisfies the communication constraints of mode 4 .

Once the matrices $P_{A}$ that define the upper-bound on the different cost-to-go values are obtained, it is possible to determine the optimal network mode for a given state.

We present next some simulations of the proposed distributed controller which can be seen as a hierarchical
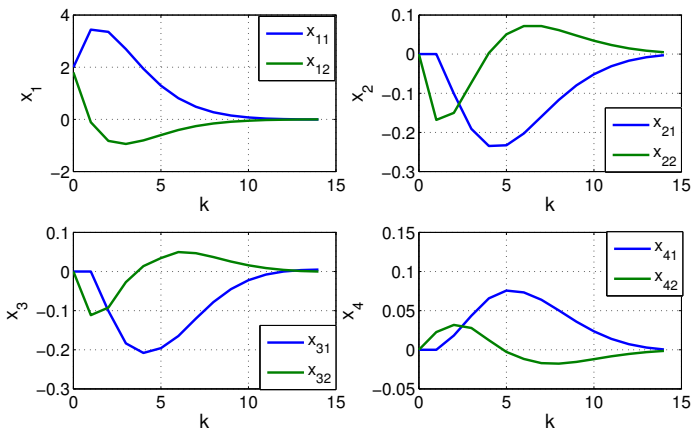

Fig. 2. States trajectories.

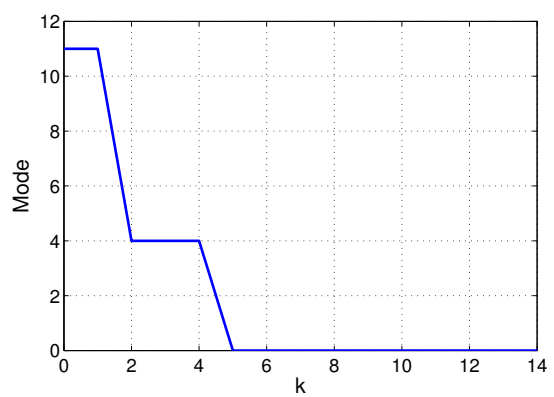

Fig. 3. Network modes.

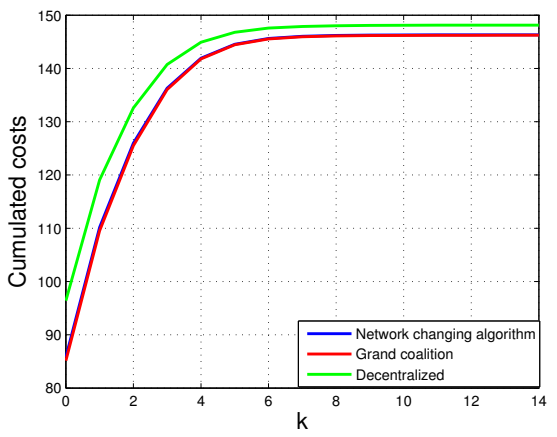

Fig. 4. Cumulated cost.

control scheme. The simulation presented here have been done with values of $D=3$ and for the initial state:

$$
x_{1}(0)=\left[\begin{array}{c}
2 \\
1.8
\end{array}\right] x_{2}(0)=\left[\begin{array}{l}
0 \\
0
\end{array}\right] x_{3}(0)=\left[\begin{array}{l}
0 \\
0
\end{array}\right] x_{4}(0)=\left[\begin{array}{l}
0 \\
0
\end{array}\right]
$$

Figures 2 shows the evolution of the system states as a function of time. Note that whenever an agents is not at the origin, it disturbs the rest of the agents from their equilibrium point. Figure 3 shows the different network modes active during the simulation. In figure 4 the cumulated cost of the coalitional distributed algorithm is compared to the cumulated cost of applying full communication at each sample. The additional communicational cost produced by the network mode choice explain the higher cost during the first steps.

\subsection{Link analysis}

Using the matrices $P_{A}$, the link game can be constructed for a given state $x$ in order to analyze which links are more relevant. The set of players for this game is defined by the 
links, which are enumerated by roman letters in figure 1. Note that the concepts of coalition of links and network mode are equivalent. For example, the characteristic function for each of the possible players of the link game for the state

$$
x_{1}=\left[\begin{array}{c}
4 \\
3.6
\end{array}\right] x_{2}=\left[\begin{array}{l}
2.1 \\
-3
\end{array}\right] x_{3}=\left[\begin{array}{l}
0.4 \\
0.8
\end{array}\right] x_{4}=\left[\begin{array}{l}
0 \\
0
\end{array}\right],
$$

is the following

$$
\begin{array}{ll}
v(I)=440.84 & v(I I)=437.61 \\
v(I I I)=439.24 & v(I V)=364.54 \\
v(I, I I)=402.93 & v(I, I I I)=439.74 \\
v(I, I V)=358.35 & v(I I, I I I)=430.53 \\
v(I I, I V)=361.81 & v(I I I, I V)=365.95 \\
v(I, I I, I I I)=354.01 & v(I, I I, I V)=354.01 \\
v(I, I I I, I V)=354.01 & v(I I, I I I, I V)=354.01 \\
v(I, I I, I I I, I V)=354.51 &
\end{array}
$$

These values show that the optimal network mode $A$ for this state is any of the four composed by three links. The Shapley value for this game is

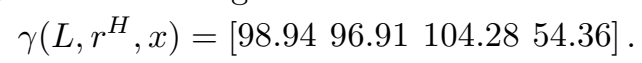

This payoff vector guarantees that the players (links) are the responsible of the costs or benefits the grand coalition gets. This implies that the higher value a link has, the less useful for the system is. It is clear that the link that is more necessary is link number $I V$, the one that connects agents 1 and 3, and for this reason this link has the lowest value. On the other hand link number $I I I$, the one that connects agents 3 and 4 , is the one that contributes less to the global objective, and so it has the highest value.

\subsection{Agent analysis}

Using the set of controllers designed using theorem 2, we evaluate the characteristic function for the agent game for state $x$ :

$$
\begin{array}{lll}
v(1)=391.12 & v(2)=35.99 & v(3)=13.22 \\
v(4)=0 & v(1,2)=427.61 & v(1,3)=328.55 \\
v(1,4)=391.12 & v(2,3)=49.21 & v(2,4)=33.26 \\
v(3,4)=12.13 & v(1,2,3)=358.35 & v(1,2,4)=389.70 \\
v(1,3,4)=329.96 & v(2,3,4)=39.40 & v(1,2,3,4)=354.01
\end{array}
$$

If we calculate the Shapley value for this game the following vector is obtained

$$
\gamma\left(N, w^{H}, x\right)=\left[\begin{array}{lll}
349.89 & 28.46-19.07-5.26
\end{array}\right] .
$$

The Shapley value is helpful from two points of view. In first place, the sum of its components adds up exactly the value that the grand coalition has assigned in the game. In cases where the characteristic function has an economic meaning this is very helpful because it provides a possible allocation vector to distribute the profits from cooperation. In second place, the Shapley value shows which agents have greater need of communication and help from their neighbors.

\section{CONCLUSIONS}

This paper contributes to the construction of a bridge between coalitional game theory and control. The main contributions of the paper are twofold. In first place, we have proposed a distributed control scheme that dynamically switches the network topology in order to optimize both the control performance and the communicational burden. In this sense, an optimization based controller design method has been provided so that all the requested matrices can be calculated. In second place, we have presented a novel interpretation of cooperative game theory tools to analyze the relevance of agents and links in a distributed control system. As it has been seen, a valuable insight into the communication structure of a distributed system is obtained from the analysis of Shapley values.

\section{REFERENCES}

A. Alessio and A. Bemporad. Decentralized model predictive control of constrained linear systems. In Proceedings of the 2007 European Control Conference, pages 28132818, 2007.

A. Alessio and A. Bemporad. Stability conditions for decentralized model predictive control under packet drop communication. In Proceedings of the 2008 American Control Conference, pages 3577-3582, 2008.

P. Borm, G. Owen, and S. Tijs. On the position value for communication situations. SIAM Journal on Discrete Mathematics, 5:305-320, 1992.

C. Canudas de Wit. Invited session on advances in networked controlled systems. In Proceedings of the 25th American Control Conference, 2006.

J. Liu, D. Muñoz de la Peña, B. Ohran, P. D. Christofides, and J. F. Davis. A two-tier architecture for networked process control. Chemical Engineering Science, 63:53495409, 2008.

J. M. Maestre, David Muñoz de la Peña, and E. F. Camacho. Distributed model predictive control based on a cooperative game. Optimal Control Applications and Methods, page In press, 2010. doi: 10.1002/oca.940.

L. Magni and R. Scattolini. Stabilizing decentralized model predictive control of nonlinear systems. Automatica, 42:1231-1236, 2006.

R. B. Myerson. Game Theory. Analysis of Conflict. Hardvard University Press, 1997.

P. Neumann. Communication in industrial automation - what is going on? Control Engineering Practice, 15: 1332-1347, 2007.

B. De Schutter R. R. Negenborn and H. Hellendoorn. Multi-agent model predictive control of transportation networks. In Proceedings of the 2006 IEEE International Conference on Networking, Sensing and Control (ICNSC 2006), 2006.

D. M. Raimondo, L. Magni, and R. Scattolini. Decentralized MPC of nonlinear system: An input-to-state stability approach. Int. J. Robust Nonlinear Control, 17:1651-1667, 2007.

W. Saad, Z. Han, M. Debbah, A. Hjorungnes, and T. Basar. Coalitional game theory for communication networks. IEEE Signal Processing Magazine, Special Issue on Game Theory, 26(5):77-97, September 2009.

A. N. Venkat. Distributed Model Predictive Control: Theory and Applications. $\mathrm{PhD}$ thesis, University of Wisconsin-Madison, 2006.

A. N. Venkat, J. B. Rawlings, and S. J. Wright. Stability and optimality of distributed model predictive control. In Proceedings of the 44th IEEE Conference on Decision and Control, and the European Control Conference ECC 2005, pages 6680-6685, Seville, Spain, 2005. 